\section{To the point}

ALTHOUGH conventionally regarded as point sources of charge, electrons may, according to some theories of highenergy physics, acquire an electric dipole - equivalent to a separation of charge. But new experiments have failed to detect any such dipole. The theories have been developed to explain a fundamental asymmetry - termed charge-parity violation - inherent in the weak nuclear force. Although different theories predict different values for the electron's dipole, it is expected to be vanishingly small. Using a 600 -fold amplification of the dipole's value expected from relativistic effects in atomic tantalum, K. Abdullah et al. (Phys. Rev. Lett. 65, 2342-2350; 1990) have sought, unsuccessfully, to find its imprint on this element's spectrum. The value the authors derive $(-2.78 \pm 8.3) \times 10^{-27} \mathrm{ecm}$ (compare water's $3.9 \times 10^{-13} \mathrm{e} \mathrm{cm}$ ) rules out more recent models invoking the increasingly popular 'Higgs boson' to explain charge-parity violation. But a further improvement of ten orders of magnitude is needed in the accuracy before all mechanisms for this asymmetry are tested.

\section{Quasi-stable}

SOME quasicrystals may be more stable than periodic crystals, claims $Z$. Olami (Phys. Rev. Lett. 65, 2559-2562; 1990). Quasicrystals have no long-range periodicity yet give sharp diffraction patterns with 'forbidden' symmetries (tenfold, for example, suggesting a quasiperiodic lattice of icosahedral symmetry). The first quasicrystals discovered, alloys of aluminium and manganese, were formed by rapid quenching, implying that they were being trapped in an uncomfortable, metastable configuration that would prefer to relax to a more stable, regular crystalline form. But by summing up interatomic interactions throughout the respective lattices, Olami finds that a monatomic face-centred icosahedral phase can be energetically more favourable than the most obvious periodic alternatives, such as body-and face-centred cubic arrays. So forming quasicrystals may prove easier than expected.

\section{Face to face}

FOLLOWING the positive identification of the skull of Wolfgang Amadeus Mozart last year, a group led by P.-F. Puech has used forensic techniques to recreate his face (L'Information Dentaire 22, 2003$2008 ; 1990)$. The skull was exhumed in 1801 , since when it has been kept in the Mozarteum in Salzburg. Reconstructing the face involves using clay to build up layers of soft tissue on a cast of the cranium. The nose is difficult to include because it depends on cartilage, but its dimensions can be calculated from the size and position of the nasal aperture only the ears are impossible to reconstruct. now $^{6}$, a $\mathrm{B}_{4} \mathrm{C} / \mathrm{Al}$ laminar composite has been made, following the abalone model, and proves to have outstanding properties (Fig. 1).

By no means was the conference limited to applications. Fundamental scientific issues were also much aired: for example, the nature of the intercrystalline boundary regions in nanostructured materials. Whereas high-resolution electron microscopy (G. Thomas and R. Siegel, Argonne National Laboratory) and Raman spectroscopy of nanocrystalline $\mathrm{CO}_{2}$ (ref. 7) indicate a normal, atomically narrow grain-boundary structure. Mössbauer studies (Gleiter; B. Fultz, California Institute of Technology), electrochemical measurements (R. Kirchheim, MaxPlanck-Institut für Metallforschung, Stuttgart, Germany), density and diffusion measurements (Gleiter) and calorimetry indicate that boundaries are more disordered and have a lower local density than conventional boundaries. The curiously sluggish grain growth already cited may be linked with this. Fultz and coworkers' Mössbauer data for a nanostructured $\mathrm{Cr}-\mathrm{Fe}$ alloy can be accurately analysed to yield an estimated boundary width of about $1 \mathrm{~nm}$; after grain growth, the boundary signal of course diminishes. The issue is still very much open; it may be that the structure depends sensitively on the nature of the misorientation and varies from boundary to boundary.

Several excellent papers were devoted to the characteristic clusters themselves. S. Riley (Argonne National Laboratory) showed how precise measurements of $\mathrm{NH}_{3}$ uptake could be used to identify the exact geometrical form of size-separated metal clusters, $\mathrm{Ni}$ and $\mathrm{Co}$ in particular, because $\mathrm{NH}_{3}$ attaches itself only to atoms standing proud of the surface, for instance at icosahedral vertices. X. Miao and $\mathrm{P}$. Marquis (Birmingham University) showed how $\mathrm{pH}$ control and the combination of two differently sized populations (of alumina and silica) allowed them to create stable combined colloid particles of the two compounds which then sinter more easily.

Magnetic studies of both elementary and multicompound nanoclusters, as well as metallic multilayers, reveal curious combinations of ferromagnetic, antiferromagnetic and superparamagnetic behaviour, as well as anomalous easy magnetization directions. In this connection, a highlight of the conference was $\mathrm{H}$. Warlimont's report on recent developments by $\mathrm{G}$. Herzer in his laboratory (Vacuumschmelze, Hanau, Germany) of an innovation first reported by Yoshizawa et al. ${ }^{8}$. Certain metallic glasses containing $\mathrm{Fe}, \mathrm{Si}$ and $\mathrm{B}$ have excellent soft magnetic properties and are currently used as transformer laminations. Yoshizawa and coworkers found that if $\mathrm{Nb}$ and (crucially) $\mathrm{Cu}$ are added to this glass and it is then

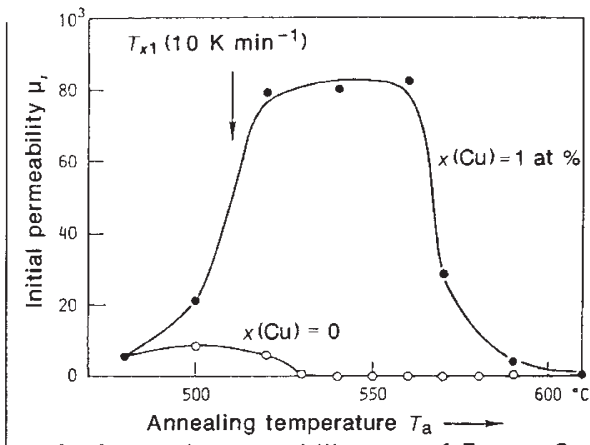

FIG. 2 Initial permeability, $\mu_{i}$, of $\mathrm{Fe}_{74.5-x} \mathrm{Cu}_{x}$ $\mathrm{Nb}_{3} \mathrm{Si}_{13.5} \mathrm{~B}_{9}$ alloys, initially glassy (with $x=0$ and $x=1$ ), as a function of annealing temperature, $T$, for a constant 1-h annealing time. $T_{x,}$ is the first crystallization temperature. (After G. Herzer and Warlimont.)

crystallized in the right way, a giant magnetic permeability can be attained, comparable with that of permalloy.

Herzer and Warlimont's results (Fig. 2) show that 1 atomic per cent of copper has a spectacular effect. It turns out that the annealed glass (provided it is not heated at too high a temperature) consists of nanocrystalline, ferromagnetic $\mathrm{Fe}_{3} \mathrm{Si}$ phase separated by films of residual glass, rich in $\mathrm{B}$ and $\mathrm{Cu}$, which is either nonmagnetic or has weaker magnetism. The residual glass crystallizes above $600{ }^{\circ} \mathrm{C}$. Herzer and Warlimont analysed the magnetic properties of such a structure.

Nanocrystalline ferromagnetic particles are single-domain (Gleiter in a recent review" cites very new evidence to this effect), and the ferromagnetic exchange interaction then overtrumps the effects of magnetocrystalline anisotrophy in the nanograins and constrains the magnetization vector to be parallel to that in a neighbouring grain, irrespective of easy directions of magnetization. At a critical grain size, this results in very low coercivity and high permeability, as observed: here the critical dimension is around $35 \mathrm{~nm}$. This $\mathrm{Cu}$-bearing alloy has also a very low magnetostriction and the hysteresis loop shape can be shaped by magnetic annealing. This kind of nanostructured magnetic dispersion in a residual glass, by analogy with the 'superstrong' dispersions cited above, might perhaps be termed a 'supermagnet' - nanostructural researchers are predisposed to superlatives.

Robert W. Cahn is in the Department of Materials Science and Metallurgy, University of Cambridge. Pembroke Street, Cambridge CB2 3QZ, UK.

1. Andres, R.P. et al. J. Mater. Res. 4, 704-736 (1989)

2. Krätschmer, W. Lamb, L.D., Fostiropoulos, K. \& Huffman D.R. Nature 347. 354-358 (1990).

Marcus, M.A. Acta metall. 27, 893-902 (1979)

4. Louat, N.P. Acta metall. 33, 59-69 (1985)

5. Wakai, F. et al Nature 344, 421-423 (1990)

6. Sarikaya, M., Gunnison, K.E., Yasrebi, M. \& Aksay, I.A. Mat. Res. Soc. Symp. Proc. 174, 109-116 (1990)

. Melendres, C. A. et al. J. Mater. Res. 4, 1246-1250 (1990).

8. Yoshizawa, Y., Oguma, S. \& Yamauchi, K. J. appl. Phys. 64, 6044 (1988)

9. Gleiter, H. Prog. Mater. Sci. 33, 1 (1990) 